\title{
SMALL-SCALE STRUCTURE OF THE MON R2 CLOUD CORE
}

\author{
TH. HENNING \\ MPG, Research Group "Dust in Star-Forming Regions", Schillergäßchen \\ 2-3, D-O-6900 Jena, Germany \\ R. CHINI \\ MPI für Radioastronomie, Auf dem Hügel 69, D-W-5300 Bonn \\ W. PFAU \\ Universitäts-Sternwarte, Schillergäßchen 2, D-O-6900 Jena
}

\section{INTRODUCTION}

High-resolution mm continuum observations are especially well suited to detect clumpy structures in molecular clouds. In this paper we concentrate on the Mon R2 cloud core which is associated with a cluster of IR sources. Walker et al. (1990) made a $1.3 \mathrm{~mm}$ map with 30" resolution. They found an unresolved and elongated structure extending from NE to SW. Here, we discuss high-resolution continuum maps at 870 and $1300 \mu \mathrm{m}$ showing a rich clumpy structure on the scale of several 10 arcsec. The clumps are probably intimately linked to the star formation process in Mon R2.

\section{OBSERVATIONS}

The MPIfR bolometer system was used at the IRAM 30-m MRT during February 1989 for mapping the Mon R2 cloud at $1300 \mu \mathrm{m}$ with a beam size of about 11 ". In September 1990 we obtained with identical equipment an $870 \mu \mathrm{m}$ map at SEST with an angular resolution of 18 ". Details of the mapping procedure, data reduction, and the results of subsequent photometry of the detected clumps are discussed elsewhere (Henning et al., 1992).

\section{SMALL-SCALE CLUMPS}

In the Fig.1a and $1 \mathrm{~b}$ we show the $870 \mu \mathrm{m}$ map and the $1300 \mu \mathrm{m}$ map. In both maps distinct dust clumps can be seen. This is especially the case in the $1300 \mu \mathrm{m}$ map. To check for coincidences, the positions of the IR sources after Hackwell et al. (1982) are also indicated. A Gaussian decomposition of the $1300 \mu \mathrm{m}$ leads to the position of seven clumps given in Table 1. IR sources are associated with five of the mm emission peaks, the identification of them is given in Table 1. The position of IR source d is after Aspin and Walther (1990), the positions of the other IR sources are after Hackwell et al. (1982). We would like to emphasize that our clump \#1 has no NIR counterpart and may be a protostellar clump. 

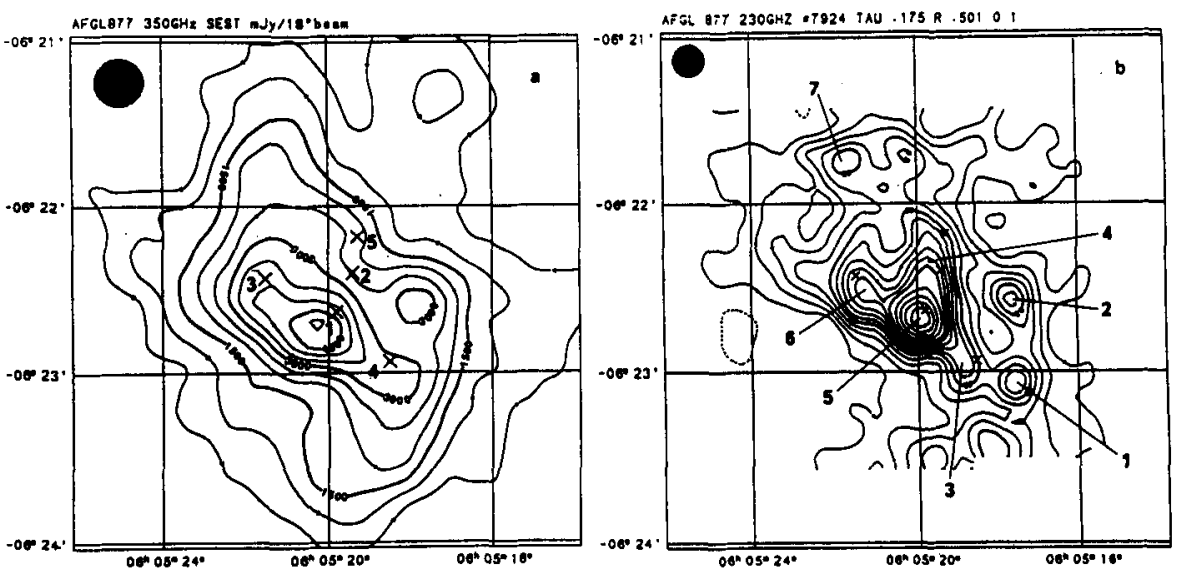

FIGURE I (a) $870 \mu \mathrm{m}$ map with 18 " resolution with the positions of the IR sources (crosses) and number after Hackwell et al. (1982) and (b) $1300 \mu \mathrm{m}$ MRT map with 11 " resolution and the location of the clumps marked by their numbers after Table 1

The clump \#7 is located outside the NIR search field of Aspin and Walther (1990).

TABLE I Millimetre clump and infrared source positions

\begin{tabular}{|c|c|c|c|c|c|}
\hline \multirow[t]{2}{*}{ number } & \multicolumn{2}{|c|}{ mm clump positions } & \multirow[t]{2}{*}{ name } & \multicolumn{2}{|c|}{ positions of infrared sources } \\
\hline & $\begin{array}{l}\mathrm{RA}(1950) \\
{[\mathrm{h} \min \mathrm{s}]}\end{array}$ & DEC(1950) & & $\begin{array}{l}\text { RA(1950) } \\
\text { h h min s] }\end{array}$ & DEC $(1950)$ \\
\hline \#1 & 060517.48 & -062303.6 & & & \\
\hline$\# 2$ & $0605 \quad 17.54$ & -062234.8 & d & 060517.3 & -062236.3 \\
\hline$\# 3$ & $06 \quad 05 \quad 18.92$ & -062256.1 & IRS 4 & 060518.5 & -062256 \\
\hline$\# 4$ & 060519.64 & -062220.8 & IRS 2 & $0605 \quad 19.4$ & -062224 \\
\hline$\# 5$ & 060519.93 & .062240 .9 & IRS 1 & 060519.8 & -062238 \\
\hline \#6 & 060521.45 & -062232.6 & IRS 3 & 060521.5 & -062226 \\
\hline$\# 7$ & 060521.84 & .062143 .7 & & & \\
\hline
\end{tabular}

\section{REFERENCES}

Aspin, C., and Walther, D.M. 1990, $A \mathcal{E} A, \mathbf{2 3 5}, 387$

Hackwell, J.A., Grasdalen, G.L., and Gehrz, R.D. 1982, ApJ, 252, 250

Henning, Th., Chini, R., and Pfau, W. 1992, $A \& A$, in press

Walker, C.K., Adams, F.C., and Lada, C.J. 1990, $A J$, 100, 1892 